\title{
Produção de mudas de melancia em diferentes ambientes e de frutos a campo ${ }^{1}$
}

\author{
Anne Mellisse Diaz Oliveira ${ }^{2}$, Edilson Costa ${ }^{* 3}$, Norton Hayd Rego ${ }^{4}$, Laís de Lima Luqui ${ }^{5}$, \\ Daniel Makoto Kusano ${ }^{6}$, Elisa Pereira de Oliveira ${ }^{7}$ \\ http://dx.doi.org/10.1590/0034-737X201562010011
}

\section{RESUMO}

A melancia ocupa lugar de destaque entre as principais olerícolas produzidas e consumidas no Brasil e, na olericultura, a produção de mudas em ambientes protegidos é a metodologia mais utilizada. O objetivo deste trabalho foi avaliar o desempenho dos cultivares de melancia "Crimson Sweet" e "Fairfax" na produção de mudas, em ambiente protegido, e de frutos, em condições de campo, na região do cerrado sul-mato-grossense. Na fase de mudas, o experimento foi conduzido em três ambientes protegidos. Cada ambiente foi considerado um experimento, conduzido no delineamento inteiramente casualizado, com seis repetições. Em seguida, realizou-se a análise conjunta. No campo, o delineamento utilizado foi o de blocos casualizados, com quatro repetições. Os dados foram submetidos à análise de variância e, as médias, ao teste de Tukey. As melhores mudas foram formadas na estufa plástica. O cultivar "Fairfax" apresentou mudas de melhor qualidade. No campo, os cultivares apresentaram frutos com diâmetros e biomassas similares.

Palavras-chave: cultivo protegido, cultivares, Citrullus lanatus Thunb. Matsum \& Nakai.

\section{ABSTRACT}

\section{Production of watermelon seedlings in different environments and fruits in the field}

Watermelon occupies a prominent position among the major vegetables produced and consumed in Brazil. Moreover, in horticulture, the seedling production in protected environments is the most used methodology. The objective of this work was to evaluate performance of "Crimson Sweet" and "Fairfax"watermelon cultivars in the production of seedlings in protected environment and of fruits in field conditions in the Brazilan Cerrado region, in the State of Mato Grosso do Sul. In the seedlings phase, the experiment was conducted in three protected environments. Each environment was regarded an experiment conducted in completely randomized design with six replicates.

After that, a joint analysis was carried out. In the field, the experimental design used was the randomized block with four replicates. The data were submitted to analysis of variance and, the means, to the Tukey's test. The best seedlings grew in the plastic greenhouse. The "Fairfax" cultivar showed higher quality seedlings. In the field, the cultivars showed fruits with similar diameter and biomass.

Key words: Citrullus lanatus (Thunb.) Matsum \& Nakai, cultivars, protected cultivation.

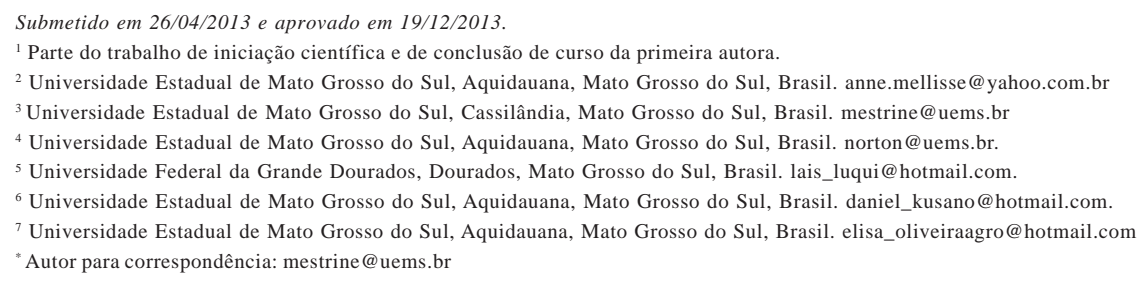




\section{INTRODUÇÃO}

A melancieira [Citrullus lanatus (Thunb.) Matsum \& Nakai.] é uma espécie de ciclo anual, originária da África e pertencente à família Cucurbitaceae. Apresenta hábito de crescimento rasteiro, com ramificações que podem alcançar de 3 a $5 \mathrm{~m}$ de comprimento. Suas raízes desenvolvem-se no sentido horizontal, concentrando-se nos primeiros 25 a $30 \mathrm{~cm}$ de profundidade do solo (Costa et al., 2010).

Por ser uma planta de clima tropical, desenvolve-se melhor em altas temperaturas, variando de 18 a $25^{\circ} \mathrm{C}$. Por outro lado, o excesso de calor, aliado à deficiência hídrica severa, pode interferir no seu desenvolvimento, causando abortamento das flores. É uma planta sensível à deficiência hídrica; portanto, durante o seu desenvolvimento deve-se ter uma boa disponibilidade de água (Lopes, 2002).

A melancia ocupa lugar de destaque entre as principais olerícolas produzidas e consumidas no Brasil, por ser cultura de fácil manejo e apresentar baixo custo de produção, sendo explorada principalmente por pequenos agricultores (Rocha, 2010).

Atualmente, o setor da horticultura vem experimentando transformações tecnológicas, com a criação de variedades cada vez mais produtivas, novos híbridos, os quais permitem um plantio de precisão, reduzindo o consumo de sementes (Zambolim et al. 2004). Segundo Rocha et al. (2010), o sucesso do cultivo de hortaliças depende da utilização de mudas de alta qualidade, aumentando a produtividade e diminuindo os riscos da produção.

Para a produção de mudas de qualidade é necessário fornecer à planta condições adequadas para o seu desenvolvimento, condições estas de nutrição, ambiência e suporte físico para o desenvolvimento da estrutura radicular. Essas condições podem ser proporcionadas às plantas a partir da utilização de ambientes protegidos para o seu cultivo (Zambolim et al., 2004). Os ambientes protegidos, dependendo do tipo de material utilizado, podem influenciar nas respostas fisiológicas (Atroch et al., 2001), como, por exemplo, o excesso de sombreamento, que causa estiolamento das mudas.

De acordo com Ferreira et al. (2006), as condições edafoclimáticas da região do Cerrado apresentam grande potencial para a produção da melancieira, sendo favorável ao desenvolvimento da planta e à qualidade dos frutos.

O objetivo deste trabalho foi avaliar a produção de mudas, em ambiente protegido, e de frutos, em condições de campo, na região do cerrado sul-mato-grossense.

\section{MATERIAL E MÉTODOS}

O experimento foi conduzido durante os meses de fevereiro a julho de 2011, na área experimental da Uni- versidade Estadual de Mato Grosso do Sul, Unidade Universitária de Aquidauana, MS, compreendida nas coordenadas geográficas de $21^{\circ} 40^{\prime} \mathrm{W}, 20^{\circ} 27^{\prime} \mathrm{S}$ e altitude de 174 metros. O solo da região é classificado como Argiloso Vermelho-Amarelo distrófico (Schiavo et al., 2010) e o clima, segundo a classificação de Koppen, é do tipo AW (tropical de savana).

Os cultivares avaliados foram "Crimson Sweet" (V1) e "Fairfax" (V2), os quais foram semeados em bandejas de 72 células, preenchidas com substrato à base de vermiculita + rama de mandioca + esterco bovino (1:1:1 em volume). Na fase de mudas, o experimento foi conduzido em três ambientes protegidos. Cada ambiente foi considerado um experimento, conduzido no delineamento inteiramente casualizado, com seis repetições de cinco plântulas cada. Em seguida, realizou-se a análise conjunta (Banzatto \& Kronka, 2006).

Foram utilizados os seguintes ambientes protegidos para a produção das mudas: a) estufa plástica (A1): estrutura de arco galvanizado com cobertura de filme de polietileno de baixa densidade (PEBD) transparente de 150 micras de espessura, abertura zenital e tela termorrefletora de $50 \%$ de sombreamento, com 8,00 m de largura por 18,00 $\mathrm{m}$ de comprimento, com altura sob a calha de 4,00 m, com tela lateral de cor preta com malha para $50 \%$ de sombreamento. Durante o experimento, a tela sob o filme permaneceu fechada (não formando sombreamento auxiliar com o filme de polietileno); b) viveiro telado, com tela na cor preta (A2): estrutura de aço galvanizado com dimensões de $8,00 \mathrm{~m}$ de largura por $18,00 \mathrm{~m}$ de comprimento, com $3,50 \mathrm{~m}$ de altura, fechamento em $45^{\circ}$, tela de monofilamento (Sombrite ${ }^{\circledR}$ ) com malha para $50 \%$ de sombreamento; c) viveiro telado com tela termorrefletora (A3): estrutura de aço galvanizado com dimensões de $8,00 \mathrm{~m}$ de largura por 18,00 m de comprimento com 3,50 m de altura, fechamento em $45^{\circ} \mathrm{s}$, com tela de aluminizada (Aluminet ${ }^{\circledR}$ ), malha para $50 \%$ de sombreamento.

A radiação global externa média diária do período foi de 404,64 W m² (estação automática de AQUIDAUANA-A719, do Instituto Nacional de Meteorologia - INMET). No presente estudo, a luz transmitida pelo polietileno de baixa densidade (plástico) para o interior do ambiente, em termos de fluxo de fótons fotossintéticos (RFA), foi aproximadamente, 551,52 $\mu \mathrm{mol} \mathrm{m} \mathrm{m}^{-2} \mathrm{~s}^{-1}$, segundo cálculo realizado a partir dos dados de radiação global externa, empregando-se a equação de Costa \& Leal (2011), definida por $\mathrm{RFA}=1,0798 * \mathrm{RGE}+114,6$, onde RGE é a radiação global externa. O cálculo foi realizado considerandose que na época do ano em que o experimento foi conduzido e para ambientes cobertos com filme de polietileno de baixa densidade, as equações de regres- 
são linear são estimativas aceitáveis na obtenção da radiação fotossinteticamente ativa em função da radiação solar global externa, e independe da orientação das casas de vegetação (Costa \& Leal, 2011).

Neste estudo, a luz transmitida pela tela preta de $50 \%$ de sombreamento para o interior do ambiente, em termos de fluxo de fótons fotossintéticos (RFA), foi de aproximadamente $470,31 \mu \mathrm{mol} \mathrm{m} \mathrm{m}^{-2} \mathrm{~s}^{-1}$, obtida pela equação de Pezzopane et al. (2004), definida por RFA = $1,97 *$ RGE. Segundo esses autores a atenuação da radiação solar provocada pela tela preta flutua ao longo do dia em função do ângulo zenital do Sol, não sendo seletiva, pois a relação entre RGE e RFA é a mesma a céu aberto e sob o ambiente protegido. Tanto internamente quanto externamente ao ambiente protegido, a relação RGE/RFA é muito similar, permitindo que mesmo tendo sido obtida em outro período do ano a equação de Pezzopane et al. (2004) possa ser aplicada no presente trabalho.

O telado com tela termorrefletora apresentou RFA interna de $335,81 \mu \mathrm{mol} \mathrm{m}{ }^{-2} \mathrm{~s}^{-1}$. Para obtenção da RFA desse ambiente, em primeiro lugar calculou-se RGI, conforme indicado por Rocha (2007), empregando-se a equação RGI $=0,544 *$ RGE. Posteriormente utilizou-se a equação de Leite (2008) que relaciona a RFA com a radiação global interna, definida por $\mathrm{RFA}=1,82 * \mathrm{RGI}$.

Foram coletadas as temperaturas $\left({ }^{\circ} \mathrm{C}\right)$ e umidades relativas $(\%)$ do ar, às 9,12 e $15 \mathrm{~h}$, dos ambientes de cultivo e externo, no período de 28 de fevereiro a 01 de abril de 2011, sendo: Externo (27,89; 82,35; 29,73; $74,08 ; 30,08 ; 72,89)$; Estufa plástica $(30,18 ; 69,17$; $31,78 ; 62,96 ; 31,37 ; 63,20)$; Viveiro telado com tela na cor preta $(28,36 ; 77,05 ; 30,59 ; 69,22 ; 31,09 ; 64,84)$ e Viveiro telado com tela termorrefletora $(27,62 ; 82,81$; 29,44; 76,42; 29,93; 73,49).

Para a preparação do substrato, o esterco bovino foi compostado por 60 dias, sendo molhado e revolvido uma vez por dia, e as ramas de mandiocas, secadas e trituradas em moinho elétrico, também foram compostadas pelo mesmo período. Foram semeadas duas sementes por célula, no dia 28 de fevereiro de 2011, na profundidade de 2 a $3 \mathrm{~cm}$. Posteriormente, foi realizado o desbaste, de maneira que foi conduzida apenas uma muda por célula. A irrigação foi realizada manualmente.

No início da fase de mudas, foram avaliados o índice de velocidade de emergência (IVE), proposto por Maguire (1962), a percentagem de emergência (PE), o tempo médio de emergência (TME), proposto por Labouriau (1983) e a velocidade média de emergência (VME) Labouriau (1970). Os dados de emergência foram coletados do dia 04 a 15 de março de 2011, com seis repetições. Para essas variáveis, cada repetição foi composta de 24 células.
Aos 31 dias após a semeadura (DAS), foram avaliados a altura da plântula (AP), o diâmetro do colo (DC), a massa de matéria seca da parte aérea (MSPA), a massa de matéria seca das raízes (MSSR), a relação massa de matéria seca aérea, massa de matéria seca radicular (RMS) e a relação altura / diâmetro do colo (RAD). Também foi determinado o índice de qualidade de Dickson (IQD) (Dickson et al., 1960). Para essas variáveis, foram utilizadas seis repetições de cinco mudas cada.

Aos 32 dias após a semeadura, no dia 02/04/2011, foi realizado o transplante para o campo. O solo local é classificado como Argissolo Vermelho distrófico (Schiavo et al., 2010), com as seguintes características na camada 0-20, obtida pela análise de solo: $\mathrm{pH}$ (água) $=$ 6,6; $\mathrm{P}=56,6 \mathrm{mg} \mathrm{dm}^{-3} ; \mathrm{K}^{+}=0,77 \mathrm{cmolc} \mathrm{dm}^{-3} ; \mathrm{Ca}^{2+}=4,4$ cmolc $\mathrm{dm}^{-3} ; \mathrm{Mg}^{2+}=1,4$ cmolc $\mathrm{dm}^{-3} ; \mathrm{SB}=6,57 \mathrm{cmolc}$ $\mathrm{dm}^{-3} ; \mathrm{H}+\mathrm{Al}^{3+}=1,6 \mathrm{cmolc} \mathrm{dm}^{-3} ; \mathrm{V}(\%)=80,42$. 64,9; em bandejas de 72

O delineamento experimental utilizado foi o de blocos completos casualizados, com quatro repetições e cinco plantas por repetição, com seis tratamentos oriundos da combinação entre ambientes e cultivares (3 ambientes X 2 cultivares $=6$ tratamentos). O espaçamento utilizado foi o de 2,0 m x 2,0 m. A irrigação foi por gotejamento. Não se realizou desbaste de frutos. O controle das plantas daninhas foi realizado por meio de capinas manuais. Foi realizada aplicação de tiametoxan na dose de $100 \mathrm{~g} \mathrm{ha}^{-1}$ (inseticida Actara $250 \mathrm{WG)} \mathrm{para} \mathrm{con-}$ trole de mosca-branca (Bemisia tabaci Gennadius). Foi realizada condução das ramas antes da frutificação para facilitar a capina.

A colheita ocorreu nos meses de junho e julho. Foram avaliados a massa e o diâmetro dos frutos, assim como o número de frutos por parcela. Para obter-se distribuição normal e homogeneidade de variâncias o número de frutos foi transformado em raiz de $(x+0,5)$. Os dados foram submetidos à análise de variância e, as médias, ao teste de Tukey, a 5\% de probabilidade, utilizando-se o software SISVAR 5.3 (Ferreira, 2010).

\section{RESULTADOS E DISCUSSÃO}

Para todas as características avaliadas, a relação entre os quadrados médios do resíduo (RQMR) das análises de variâncias individuais dos experimentos não ultrapassou o valor de 7:1, permitindo, portanto, a realização da análise conjunta dos experimentos (Banzatto \& Kronka, 2006) e a comparação dos ambientes protegidos.

$\mathrm{Na}$ interação entre ambiente e cultivar verificou-se diferença estatística significativa para as características MSPA, MSSR, MST e IQD (Tabela 1), não sendo observada diferença significativa para as demais característi- 
cas (IVE, PE, TME, VME - Tabela 2; AP, DC, RAD e RMS -Tabela 3).

As mudas do cultivar "Fairfax" apresentaram os maiores valores para as variáveis MSPA, MSSR, MST e IQD (Tabela 1). O Índice de Qualidade de Dickson (IQD) é utilizado como fator de qualidade da muda e, quanto maior o seu valor, maior é o padrão de qualidade das mudas. Por isso, pode-se afirmar que as mudas do cultivar "Fairfax", que apresentaram IQD = 0,052, produzidas nestas condições, foram melhores do que as do cultivar "Crimson Sweet", que apresentaram IQD = 0,025. O Índice de qualidade de Dickson (IQD) foi um bom indicador de qualidade de mudas de melancia, pois abrange diversas características que tiveram o mesmo comportamento, especialmente as produções de biomassa (MSPA, MSSR e MST).

Para ambos os cultivares, a estufa agrícola propiciou melhores condições que os telados, promovendo plantas com maiores biomassas e com maior IQD (Tabela 1). A maior adaptabilidade dos cultivares a esse ambiente, em relação aos demais, pode estar relacionada com o menor sombreamento neste ambiente, fazendo com que não ocorresse estiolamento e, com isso, um menor gasto de energia, favorecendo a formação de mudas de melhor qualidade. Pela estimativa da RFA, esse ambiente também apresentava a maior disponibilidade de fluxo de fótons fotossintéticos $\left(551,52 \mu \mathrm{mol} \mathrm{m} \mathrm{m}^{-2} \mathrm{~s}^{-1}\right)$, favorecendo o melhor desenvolvimento das mudas.

Para as características IVE, PE e VME, a estufa plástica e o Sombrite ${ }^{\circledR}$ não apresentaram diferenças significativas entre si, sendo seus valores superiores aos do viveiro telado Aluminet ${ }^{\circledR}$ (Tabela 2). Em seu trabalho, avaliando o efeito do ambiente sobre mudas de maracujazeiro-amarelo, Costa et al. (2009) observaram que o ambiente Sombrite ${ }^{\circledR}$ proporcionou melhores resultados que a estufa e o Aluminet ${ }^{\circledR}$, para o desenvolvimento das mudas.

Os menores índices de emergência, observados no ambiente com tela termorrefletora (Aluminet ${ }^{\circledR}$ ), podem estar relacionados com temperaturas possivelmente inferiores às dos demais ambientes, ao final da tarde e início da noite. Este tipo de ambiente poderia estar armazenando menos energia térmica para o período noturno, desfavorecendo o processo de emergência. Pezzopane et al. (2004) afirmaram que as telas termorrefletoras contribuem para a diminuição da transpiração da cultura à noite e, consequentemente, para a redução do calor consumido por evapotranspiração. No entanto, na fase inicial de emergência das plântulas, essas características das telas não auxiliaram o desenvolvimento, pois as plantas, nessa fase, ainda não apresentam suas folhas definitivas.

Tabela 1. Interações entre ambientes e cultivares para a fitomassa seca da parte aérea (MSPA), fitomassa seca do sistema radicular (MSSR), fitomassa seca total (MST) e índice de qualidade de Dickson (IQD) da melancieira, cultivares Crimson Sweet e Fairfax

\begin{tabular}{|c|c|c|c|c|c|c|}
\hline & Estufa & Sombrite $^{\circledR}$ & Aluminet $^{\circledR}$ & Estufa & Sombrite $^{\circledR}$ & Aluminet $^{\circledR}$ \\
\hline & \multicolumn{3}{|c|}{ MSPA } & \multicolumn{3}{|c|}{ MSSR } \\
\hline Crimson Sweet & $0,143 \mathrm{Ba}$ & $0,111 \mathrm{Bb}$ & $0,120 \mathrm{Aab}$ & $0,028 \mathrm{Ba}$ & $0,015 \mathrm{Bb}$ & $0,016 \mathrm{Bb}$ \\
\hline Fairfax & $0,244 \mathrm{Aa}$ & $0,158 \mathrm{Ab}$ & $0,131 \mathrm{Ac}$ & $0,054 \mathrm{Aa}$ & $0,033 \mathrm{Ab}$ & $0,023 \mathrm{Ac}$ \\
\hline \multirow[t]{2}{*}{$\mathrm{CV}(\%)$} & 10,9 & & & \multicolumn{3}{|l|}{16,7} \\
\hline & \multicolumn{3}{|c|}{ MST } & \multicolumn{3}{|c|}{ IQD } \\
\hline Crimson Sweet & $0,171 \mathrm{Ba}$ & $0,126 \mathrm{Bb}$ & $0,136 \mathrm{Ab}$ & $0,025 \mathrm{Ba}$ & $0,013 \mathrm{Bb}$ & $0,014 \mathrm{Bb}$ \\
\hline Fairfax & $0,298 \mathrm{Aa}$ & $0,190 \mathrm{Ab}$ & $0,154 \mathrm{Ac}$ & $0,052 \mathrm{Aa}$ & $0,029 \mathrm{Ab}$ & $0,021 \mathrm{Ac}$ \\
\hline $\mathrm{CV}(\%)$ & 10,6 & & & 15,0 & & \\
\hline
\end{tabular}

* Letras iguais maiúsculas nas colunas e minúsculas nas linhas, para cada variável, não diferem entre si pelo teste de Tukey a 5\% de probabilidade. $\mathrm{CV}=$ coeficiente de variação.

Tabela 2. Índice de velocidade de emergência (IVE), percentagem de emergência (PE), tempo médio de emergência (TME) e velocidade média de emergência (VME) da melancieira, cultivares Crimson Sweet e Fairfax

\begin{tabular}{lcccc}
\hline & IVE & PE & TME & VME \\
\hline Estufa (A1) & $23,9 \mathrm{~A}$ & $79,9 \mathrm{~A}$ & $10,0 \mathrm{~B}$ & $0,1003 \mathrm{~A}$ \\
Sombrite $^{\circledR}$ (A2) & $21,0 \mathrm{~A}$ & $74,3 \mathrm{~A}$ & $10,1 \mathrm{~B}$ & $0,0990 \mathrm{~A}$ \\
Aluminet $^{\circledR}$ (A3) & $15,5 \mathrm{~B}$ & $60,1 \mathrm{~B}$ & $10,3 \mathrm{~A}$ & $0,0967 \mathrm{~B}$ \\
Crimson Sweet (V1) $^{\text {Fairfax (V2) }}$ & $26,8 \mathrm{a}$ & $91,0 \mathrm{a}$ & $10,0 \mathrm{~b}$ & $0,1001 \mathrm{a}$ \\
\hline CV $(\%)$ & $13,5 \mathrm{~b}$ & $51,9 \mathrm{~b}$ & $10,3 \mathrm{a}$ & $0,0973 \mathrm{~b}$ \\
\hline
\end{tabular}

*Letras iguais maiúsculas nas colunas para os ambientes protegidos, assim como letras minúsculas para os cultivares de melancia, para cada variável, não diferem entre si pelo teste de Tukey a $5 \%$ de probabilidade. CV = coeficiente de variação. 
Estes aspectos de energia térmica acumulada, em função da época do ano em que o experimento foi realizado, podem ter contribuído para que o Sombrite ${ }^{\circledR}$ e a estufa plástica propiciassem melhores condições para emissão da radícula da semente e, portanto, melhores condições de emergência.

Para a variável tempo médio de emergência (TME), a estufa plástica apresentou os menores valores, mostrando-se superior (Tabela 2). Neste trabalho, a duração média da emergência foi de dez dias, resultado maior que o observado por Belfort et al. (2003), que encontraram duração de seis dias, com o cultivar 'Nova", híbrido triploide, em ambiente telado de cor preta.

O cultivar "Crimson Sweet" teve percentagem de emergência $(\mathrm{PE}=91 \%)$ maior que a do cultivar Fairfax $(\mathrm{PE}=51,9 \%)$. Este resultado é inferior ao de Bhering et al. (2003), que obtiveram 97,5\% com "Crimson Sweet". Além da percentagem de emergência, o cultivar "Crimson Sweet" também apresentou melhores resultados para as demais características avaliadas (IVE, TME e VME - Tabela 2). Peixoto \& Matta (2010) também verificaram desempenho superior do cultivar de melancia "Crimson Sweet" em relação aos cultivares Sugar Baby, Omaru Yanato e Fairlax para o índice de velocidade de emergência.

Quanto às características AP e RMS, o viveiro telado Aluminet $^{\circledR}$ e o viveiro Sombrite ${ }^{\circledR}$ não se diferenciaram entre si, porém, foram superiores à estufa plástica para essas características (Tabela 3). As maiores alturas ob- servadas nas plantas cultivadas no ambiente recoberto com Sombrite ${ }^{\circledR}$ podem estar relacionadas com a percentagem de sombreamento e a cor da tela, ocorrendo um possível início de estiolamento das mudas, expressado pela RAD, uma vez que nesse ambiente, o diâmetro do colo foi menor do que nas plantas dos demais ambientes (Tabela 3).

Quanto às variáveis altura de plântula (AP), diâmetro do colo (DC) e relação das massas de matéria seca das partes aérea e radicular (RMS), o cultivar "Crimson Sweet" apresentou resultados que denotam inferioridade em relação ao "Fairfax", na fase de formação de mudas. Esses dados revelam que o cultivar "Crimson Sweet" é mais sensível ao sombreamento, pois apresentou tendência ao estiolamento, conforme verificado pela RAD (Tabela 3).

Houve baixa produção de frutos, com frutos de dimensões e pesos pequenos. Não foram observadas diferenças para as características dos frutos, nos seis tratamentos (ambientes x cultivares) testados neste experimento (Tabela 4). O peso médio de frutos é das características que apresentam maior variação, quando se trabalha com diferentes grupos de cultivares. Contudo, não foi verificada essa diferença entre os dois cultivares avaliados.

As massas do fruto obtidas, neste trabalho, de 1,96 kg, para "Crimson Sweet", e de 1,59, para "Fairfax", foram inferiores às observadas por Ramos et al. (2009), que obtiveram média de $6,33 \mathrm{~kg}$ por fruto de "Crimson

Tabela 3. Altura de plântula (AP), diâmetro do colo (DC), relação altura e diâmetro do colo (RAD) e relação fitomassa seca aérea e radicular (RMS) da melancieira, cultivares Crimson Sweet e Fairfax

\begin{tabular}{lcccc}
\hline & AP & DC & RAD & RMS \\
\hline Estufa (A1) & $4,26 \mathrm{~B}$ & $2,77 \mathrm{~A}$ & $1,55 \mathrm{C}$ & $4,84 \mathrm{~B}$ \\
Sombrite $^{\circledR}$ (A2) & $5,26 \mathrm{~A}$ & $2,43 \mathrm{~B}$ & $2,21 \mathrm{~A}$ & $6,23 \mathrm{~A}$ \\
Aluminet $^{\circledR}$ (A3) & $5,28 \mathrm{~A}$ & $2,68 \mathrm{~A}$ & $1,98 \mathrm{~B}$ & $6,75 \mathrm{~A}$ \\
Crimson Sweet (V1) $_{\text {Fairfax (V2) }}^{5,60 \mathrm{a}}$ & $2,51 \mathrm{~b}$ & $2,25 \mathrm{a}$ & $6,84 \mathrm{a}$ \\
\hline CV $(\%)$ & $4,27 \mathrm{~b}$ & $2,74 \mathrm{a}$ & $1,57 \mathrm{~b}$ & $5,04 \mathrm{~b}$ \\
\hline
\end{tabular}

*Letras iguais maiúsculas nas colunas para os ambientes protegidos, assim como letras minúsculas para os cultivares de melancia, para cada variável, não diferem entre si pelo teste de Tukey a $5 \%$ de probabilidade. CV = coeficiente de variação.

Tabela 4. Características dos frutos de melancieira, cultivares Crimson Sweet e Fairfax

\begin{tabular}{lccc}
\hline & Massa (kg) & Diâmetro (cm) & Número \\
\hline Estufa/ Crimson Sweet & $2,34 \mathrm{~A} *$ & $15,62 \mathrm{~A}$ & $3,80 \mathrm{~A}$ \\
Estufa/ Fairfax & $1,59 \mathrm{~A}$ & $12,95 \mathrm{~A}$ & $5,00 \mathrm{~A}$ \\
Sombrite $^{\circledR} /$ Crimson Sweet & $1,96 \mathrm{~A}$ & $15,29 \mathrm{~A}$ & $4.80 \mathrm{~A}$ \\
Sombrite $^{\circledR} /$ Fairfax & $2,06 \mathrm{~A}$ & $12,35 \mathrm{~A}$ & $4,00 \mathrm{~A}$ \\
Aluminet $^{\circledR} /$ Crimson Sweet & $2,13 \mathrm{~A}$ & $14,76 \mathrm{~A}$ & $4,80 \mathrm{~A}$ \\
Aluminet $^{\circledR} /$ Fairfax & $2,90 \mathrm{~A}$ & $15,67 \mathrm{~A}$ & $3,30 \mathrm{~A}$ \\
Coeficiente de Variação $(\%)^{\text {Erro padrão da média }}$ & 26,49 & 13,92 & 25,48 \\
\hline
\end{tabular}

*Letras iguais maiúsculas nas colunas, para cada variável, não diferem entre si pelo teste de Tukey a 5\% de probabilidade. 
Sweet". Garcia \& Sousa (2002), trabalhando com "Crimson Sweet", observaram que, na medida em que aumentava a população de plantas, ocorria diminuição nos pesos médios de frutos.

A produção média do cultivar "Crimson Sweet" correspondeu a uma produtividade 4377,3 $\mathrm{kg} \mathrm{ha}^{-1}$ e do cultivar "Fairfax" correspondeu a 3259,5,3 kg ha-1 Estes resultados mostraram baixa produtividade quando comparados aos de Ramos et al. (2009) que verificaram $86580 \mathrm{~kg}$ para o cultivar "Crimson Sweet" no espaçamento médio de 2,0 x 0,4 m. Entretanto, deve-se considerar que neste experimento o espaçamento de 2,0 x 2,0 m. Segundo Pedrosa (2007) a produtividade média da melancia se situa entre 20000 e $25000 \mathrm{~kg}$ por hectare, o que teria sido alcançado neste estudo se usado o espaçamento de $2,0 \times 0,4 \mathrm{~m}$.

\section{CONCLUSÕES}

As melhores mudas foram formadas no ambiente de estufa plástica;

O cultivar "Fairfax" apresentou mudas de melhor qualidade;

No campo, os cultivares apresentaram frutos com diâmetros e biomassas similares.

\section{AGRADECIMENTOS}

Ao CNPq Proc. No 300829/2012-4; à FUNDECT Proc. No 23/200.647/2012; e à PROPP/UEMS.

\section{REFERÊNCIAS}

Atroch EMAC, Soares AM, Alvarenga AA \& Castro EM (2001) Crescimento, teor de clorofilas, distribuição de biomassa e características anatômicas de plantas jovens de Bauhinia forticata LINK submetidas a diferentes condições de sombreamento. Ciência e Agrotecnologia, 25:853-862.

Banzatto DA \& Kronka SN (2006) Experimentação agrícola. $3^{\mathrm{a} e d .}$ Jaboticabal, Funep. 237p.

Belfort CC, Nery EB, CAvalcante JIV, Thé FW, Silva Filho JAS \& Setúbal JW (2003) Influência do processo de semeadura na emergência de plântulas de melancia triplóide. In: Congresso Brasileiro de Olericultura, Recife. Resumos, Sociedade Brasileira de Olericultura. 4p.

Bhering MC, Dias DCFS, Barros DI, Dias LASD \& Tokuhisa D (2003) Avaliação do vigor de sementes de melancia (Citrullus lanatus Schrad.) pelo teste de envelhecimento acelerado. Revista Brasileira de Sementes, 25:1-6.

Costa ARFC, Medeiros JF, Porto Filho FQ, Silva JS, Freitas DC \& Costa FGB (2010) Produção de cultivares de melancia submetidas a níveis de salinidade da água de irrigação e doses de nitrogênio. Revista Brasileira de Agricultura Irrigada, 4:242-248.

Costa E, Rodrigues ET, Alves VB, Santos LCR \& Vieira LCR (2009) Efeitos da ambiência, recipientes e substratos no desenvolvimento de mudas de maracujazeiro-amarelo em Aquidauana - MS. Revista Brasileira de Fruticultura, 31:236-244.
Costa E \& Leal PAM (2011) Medidas radiométricas em casas de vegetação com cobertura plástica na região de Campinas-SP. Engenharia Agrícola, 31:448-457.

Dickson A, Leaf AL \& Hosner JF (1960) Quality appraisal of white spruce and white pine seedling stock in nurseries. Forestry Chronicle, 36:10-13.

Ferreira DF (2010) Sistema de análise de variância. Versão 5.3. Lavras, UFLA. 66p.

Ferreira MAJF, Queiróz MA, Vencovsky R \& Duarte JB (2006) Prémelhoramento de uma população de melancia com sistema misto de reprodução. Pesquisa Agropecuária Tropical, 36:131-139.

Garcia LF \& Souza VAB (2002) Influência do espaçamento e da adubação nitrogenada sobre a produção da melancia. Revista de la Facultad de Agronomia, 28:59-70.

Labouriau LG (1983) A germinação de sementes. Washington: Organização dos Estados Americanos (OEA), Programa Regional de Desenvolvimento Científico e Tecnológico. Washington, Secretaria Geral da OEA.174p.

Labouriau LG (1970) On the physiology of seed germination in Vicia graminea Sm. Anais da Academia Brasileira de Ciências, 42:235-262.

Leite MCO (2008) Uso de malhas termo-refletoras e malha negra no cultivo hidropônico da alface. Dissertação de Mestrado. Mossoró, Universidade Federal Rural do Semi-Árido. 39p.

Lopes CRB (2002) Avaliação de cultivares de melancia na depressão central do Rio Grande do Sul. Dissertação de mestrado. Porto Alegre, Universidade Federal do Rio Grande do Sul. 55p.

Maguire JD (1962) Speed of germination aid in selection and evaluation of seedling emergence and vigor. Crop Science, 2:176-177.

Pedrosa JF (1997) Cultura da melancia. 2. ed. Mossoró, Escola Superior de Agricultura de Mossoró (ESAM). 53 p.

Peixoto LA \& Matta FP (2010) Avaliação de genótipos de melancia quanto as suas características de germinação e vigor. In: Encontro Latino Americano de Iniciação Científica, Alegre. Resumos, Universidade do Vale do Paraíba. 4p.

Pezzopane JEM, Oliveira PC, Reis EF \& Lima JSS (2004) Alterações microclimáticas causadas pelo uso da tela plástica. Engenharia Agrícola, 24:9-15.

Ramos ARP, Dias RCS \& Aragão CA (2009) Densidades de plantio na produtividade e qualidade de frutos de melancia. Horticultura Brasileira, 27:560-564.

Rocha RC (2007) Uso de diferentes telas de sombreamento no cultivo protegido do tomateiro. Dissertação de mestrado. Universidade Estadual Paulista "Júlio De Mesquita Filho", Botucatu. 90p.

Rocha MR (2010) Sistemas de cultivo para a cultura da melancia. Dissertação de mestrado. Universidade Federal de Santa Maria, Santa Maria. 76p.

Schiavo JA, Pereira MG, Miranda LPM, Dias Neto AH \& Fontana A (2010) Caracterização e classificação de solos desenvolvidos de arenitos da formação Aquidauana - MS. Revista Brasileira de Ciência do Solo, 34:881-889.

Zambolim L, Vida JB, Tessmann DJ, Brandão Filho JUT, Verzignassi JR \& Caixeta MP (2004) Manejo de doenças de plantas em cultivo protegido. Fitopatologia Brasileira, 29:355-372. 